\title{
Quantitative profiling of regional protein expression in rat retina after partial optic nerve transection using fluorescence difference two-dimensional gel electrophoresis
}

\author{
CHUEN LAM ${ }^{1 *}$, KING KIT LI ${ }^{1}$, CHI WAI DO ${ }^{1}$, HENRY CHAN ${ }^{1}$, CHI HO TO ${ }^{1}$ and JACKY MAN KWONG KWONG ${ }^{2 *}$ \\ ${ }^{1}$ School of Optometry, Hong Kong Polytechnic University, Hong Kong 999077, SAR, P.R. China; \\ ${ }^{2}$ Department of Ophthalmology, Stein Eye Institute, David Geffen School of Medicine, \\ University of California Los Angeles, Los Angeles, CA 90095, USA
}

Received February 8, 2019; Accepted May 14, 2019

DOI: $10.3892 / \mathrm{mmr} .2019 .10525$

\begin{abstract}
To examine the difference between primary and secondary retinal ganglion cell (RGC) degeneration, the protein expression at four regions of retina including superior, temporal, inferior and nasal quadrant in a rat model of partial optic nerve transection (pONT) using 2-D Fluorescence Difference Gel Electrophoresis (DIGE) were investigated. Unilateral pONT was performed on the temporal side of optic nerves of adult Wistar rats to separate primary and secondary RGC loss. Topographical quantification of RGCs labeled by Rbpms antibody and analysis of axonal injury by grading of optic nerve damage at 1 week $(n=8)$ and 8 weeks $(n=15)$ after pONT demonstrated early RGC loss at temporal region, which is considered as primary RGC degeneration and progressing RGC loss at nasal region, which is considered as secondary RGC degeneration. Early protein expression in each retinal quadrant $(n=4)$ at 2 weeks after pONT was compared with the corresponding quadrant in the contralateral control eye by DIGE. For all comparisons, 24 differentially expressed proteins ( $>1.2$-fold; $\mathrm{P}<0.05 ; \geq 3$ non-duplicated peptide matches) were identified by mass spectrometry (MS). Interestingly, in the nasal retina, serum albumin and members of crystallin family, including $\alpha \mathrm{A}, \alpha \mathrm{B}, \beta \mathrm{A} 2, \beta \mathrm{A} 3, \beta \mathrm{B} 2$ and $\gamma \mathrm{S}$ indicating stress response were upregulated. By contrast, only $\alpha \mathrm{B}$ and $\beta \mathrm{A} 2$ crystallin proteins were altered in temporal quadrant. In the superior and inferior quadrants, $\beta \mathrm{B} 2$ crystallin, keratin type I, $\mathrm{S}$-arrestin and lamin-B1 were upregulated, while heat shock
\end{abstract}

Correspondence to: Dr Jacky Man Kwong Kwong, Department of Ophthalmology, Stein Eye Institute, David Geffen School of Medicine, University of California Los Angeles, 100 Stein Plaza, Los Angeles, CA 90095, USA

E-mail:kwong@jsei.ucla.edu

*Contributed equally

Key words: retinal ganglion cell, proteomics, neurodegeneration, optic nerve, retina, protein cognate $71 \mathrm{kDa}$ protein and heterogeneous nuclear ribonucleoproteins A2/B1 were downregulated. In summary, the use of DIGE followed by MS is useful to detect early regional protein regulation in the retina after localized optic nerve injury.

\section{Introduction}

In the central nervous system (CNS), the final extent of functional impairment following ischemic and traumatic injury depends on not only how severe is the damage caused by the primary lesion but also which chemicals are released and, in turn, how they stimulate chain reactions and mediate responses in the adjacent areas, while the disease or injury is spreading (1,2). Mostly, secondary degeneration processes take place in the neurons and glia away from the primary injury site, which initially remain intact and functional but may die at later time (3-5). For example, glaucomatous optic neuropathy involves injury of retinal ganglion cell (RGC) axons at a localized region of the optic nerve frequently to mechanical insult induced by intraocular pressure (IOP) elevation $(6,7)$. Subsequently, secondary RGC degeneration leads to widespread loss of vision over an extended period of time (8). To develop therapies to halt this progressive damage and preserve function, understanding the mechanism of secondary degeneration and the responses of the neural tissue is essential (9).

It is challenging to study primary and secondary RGC degeneration separately in glaucomatous or traumatic optic neuropathy. A partial optic nerve model has been developed to distinguish secondary degeneration from primary RGC degeneration morphologically, as well as their temporal and spatial variation $(10,11)$. An area of the retina that is expected to demonstrate dramatic loss of RGCs less than two weeks after the partial transection or direct trauma to the optic nerve. This process is considered as primary degeneration. In other retinal areas, locations beyond the initial injury site, delayed and gradual loss of RGCs occurs after some weeks and this process is considered as secondary degeneration (12). Investigations of secondary degeneration have showed that RGCs die by apoptosis as a result of activation of proteins of the bcl-2 family and caspase families and the release of reactive oxygen species (ROS) from the transected site which 
is proposed to be a key upstream pathway leading to the cell death cascades (13-15). Differences between the pathways for primary and secondary RGC degeneration and differential drug effects have been recognized (14-16). However, the exact factors contributing to the death of the non-transected RGCs or whether any protective mechanism can counteract the damaging insults remains to be elucidated $(17,18)$. An investigation of the regional expression of proteins may help provide an understanding of the specific pathway leading to secondary RGC degeneration. In this study, by utilizing the partial optic nerve injury model, which separates primary and secondary degeneration, it is possible to evaluate the degree of damage both the RGC axon in the optic nerve and the RGC body in the retina, and to locate the sites of primary and secondary RGC degeneration. To explore the differences between protein expressions at different locations, the retina was divided into 4 quadrants for the investigation. To determine whether the retinal proteins were regulated during primary or secondary RGC degeneration two-dimensional fluorescence difference gel electrophoresis (DIGE) was performed and the identities of differentially expressed proteins were confirmed by tandem mass spectrometry (MS).

\section{Materials and methods}

Animals. The use of animals in the study was approved by the Animal Research Committee of the University of California, Los Angeles. The procedures were performed in compliance with the ARVO Statement for the Use of Animals in Ophthalmic and Vision Research. Three month old male Wistar rats weighting 300-350 g were housed with standard food and water provided ad libitum in animal research facility of the University of California Los Angeles. Lighting was turned on at $3 \mathrm{am}$ and off at $3 \mathrm{pm}$. The animals were kept for at least one week in this environment before surgical procedures. Animals were sacrificed by carbon dioxide overdose at various time points after pONT with cervical dislocation as secondary euthanasia method to ensure the complete euthanasia.

Partial optic nerve transection ( $p O N T$ ). Previously published procedures for pONT were modified and performed in animals anesthetized with isoflurane gas and topical proparacaine $1 \%$ eye drops (11). An incision was made in the temporal conjunctiva rather than the superior conjunctiva due to easier access to the nerve behind the globe without excessive retraction of the globe. A diamond knife for radial keratotomy was used to incise the optic nerve to a depth of one third of its diameter 2-3 mm behind the eye. A metal guard limited penetration and variation of incision distance. Prior to the procedure, the diameter of control optic nerves of adult Wistar rats $(n=20)$ from histological optic nerve cross sections previously collected was estimated. The averaged diameter was found to be approximately $0.87 \mathrm{~mm}$ and, therefore, the depth of the blade was adjusted to $0.28 \mathrm{~mm}$. A partial cross section of the optic nerve was made carefully, so as not to damage the adjacent blood supply. All eyes were examined ophthalmoscopically to ensure complete retinal blood flow. The conjunctival incision was sutured and topical ophthalmic ointment (tobramycin, Tobrex; Alcon, Fort Worth, TX, USA) was applied immediately after the surgical procedures and then twice daily for
2 days. Surgical procedures were performed on one eye of each rat, the contralateral eye serving as an untreated control.

Experimental design. To evaluate primary and secondary RGC loss after pONT, 30 animals were included and randomly divided into three groups: Normal $(n=10), 1$ week $(n=8)$ and 8 weeks $(n=12)$. Immunohistochemistry using Rbpms antibody and topographical quantification of RGCs in the retinal wholemount was performed, while the optic nerve segments 1-2 mm behind the globe were collected for the grading of the injury. To explore early differentially expressed proteins after pONT, an additional 4 animals (numbers: 1824, 1825, 1826 and 1827) were sacrificed 2 weeks after pONT. Each retina was carefully and equally divided into 4 quadrants including superior, temporal, inferior, and nasal under a dissection microscope, frozen immediately in liquid nitrogen, and analyzed separately using a DIGE approach according to our published protocol (19). The protein candidates with significant changes were identified using tandem MS/MS mass spectrometry.

Rbpms immunohistochemistry on retinal wholemounts and $R G C$ quantification. Animals were deeply anesthetized with intramuscular injections of $80 \mathrm{mg} / \mathrm{kg}$ sodium pentobarbital and then transcardially perfused with $4 \%$ paraformaldehyde in $0.1 \mathrm{M}$ phosphate buffer. After enucleation and post-fixation for $1 \mathrm{~h}$, the retinas were dissected and processed with anti-Rbpms antibodies as described previously (20). Briefly, all retinas were bisected for better staining results than for the whole retina methods. The samples were incubated with $10 \%$ fetal bovine serum for $1 \mathrm{~h}$ to block nonspecific staining, and then immersed in Rbpms antibody in PBS containing 1\% Triton, $0.5 \%$ BSA, and $0.9 \%$ sodium chloride (PBS-T-BSA) overnight at $4{ }^{\circ} \mathrm{C}$. After washing in PBS-T-BSA, the retinas were incubated with secondary Alexa Fluor 488 goat anti-rabbit IgG antibody $(1 / 1,000)$ overnight at $4^{\circ} \mathrm{C}$. With radial cuts, the retinas were mounted on a glass slide and air dried. Topographical analysis of immunolabeled cells was performed as previously described $(20,21)$. The percentage of cell loss was defined as the decreased number of cells in the experimental eye as a percentage of the density in the contralateral control eye of the same animal.

Optic nerve injury analysis. To semi-quantify the axonal injury, a reliable method of grading optic nerve injury was adopted $(22,23)$. The tissues were fixed, processed, and embedded in acrylic resin. One-micrometer-thick sections were cut and stained with $1 \%$ toluidine blue. The areas of the optic nerve cross sections were measured by Image $\mathbf{J}$ software (NIH, Bethesda, MD, USA). Each section was manually divided into two areas (transected and non-transected) for grading separately. Optic nerve injury was assessed by two independent masked observers using a graded scale ranging from 1 (normal; no degenerated axon was noted) to 5 (total degeneration; all axons showed degenerated organelles, axonal content, and myelin sheath).

Homogenization and protein extraction. Each frozen retinal sample was homogenized with $100 \mu$ lysis buffer containing $30 \mathrm{mM}$ Tris- $\mathrm{HCl}$ (pH 8.5), $7 \mathrm{M}$ urea, $2 \mathrm{M}$ thiourea, $2 \%$ CHAPS, $1 \%$ ASB14 and Complete, Mini protease inhibitor (1 tablet per 
$10 \mathrm{ml}$ buffer) in a liquid nitrogen cooled Teflon freezer mill (Mikro-Dismembrator Braun Biotech, Melsungen, Germany). A tungsten carbide $(9 \mathrm{~mm})$ grinding ball was placed inside the chamber to help shatter the tissue into powder form at low temperature during dismembration. The homogenate was incubated for $30 \mathrm{~min}$ on ice and then centrifuged at $16,100 \mathrm{~g}$ for $20 \mathrm{~min}$ at $4^{\circ} \mathrm{C}$. The supernatant was collected, and protein concentration determined by PlusOne 2-D Quant kit (GE Healthcare, Life Science, Marlborough, MA, USA).

Protein sample labeling with CyDyes ${ }^{T M}$ for DIGE. Lysine labeling protocol, which referred to as 'minimal labeling' was utilized in this study. As previously described $(19,24)$, soluble protein lysates from each specific retinal region (Superior/Temporal/Inferior/Nasal) of the treated and control eyes were randomly labeled with either Cy3 or Cy5 Dye. An additional pooled protein lysate (a mixture of equal amounts of proteins from all the four regions of both the treated and control eyes) was labeled with Cy 2 as an internal standard. To avoid the bias from dye labeling efficiency, dye-swapping was adopted in the treated and control retina.

Two-dimensional gel electrophoresis \& image analysis. Isoelectric focusing (IEF) was performed using linear immobilised $\mathrm{pH}$ gradient (IPG) strips (17 cm, pH 3-10 NL) as previously described (19). The Cy2, Cy3, and Cy5-labeled images were acquired on a Typhoon model 9400 Variable Mode Imager (GE Healthcare, Life Science) at excitation/emission values of 488/520, 532/580, 633/670 nm respectively with a resolution of $100 \mu \mathrm{m}$. After scanning, 2D gels were fixed and further stained with MS compatible silver stain for spot visualization $(19,24,25)$. The images of the scanned gels were cropped using the ImageQuant ${ }^{\mathrm{TM}} \mathrm{V} 5.0$ to remove extraneous areas of the gel images. Image analysis was then performed using DeCyder Differential Analysis Software, version 6.0 (Amersham Biosciences Corp.). Spot detection and quantification were performed using the differential in-gel analysis (DIA) mode and images from different gels were matched using the biological variance analysis (BVA) mode so pair-wise image analysis among all gels could be performed. The automatic matching of protein spots across different gels was further confirmed by manual scanning to avoid potential artifacts. Protein spots with an expression change $>1$. 2 -fold and Student's Paired t-test, $\mathrm{P} \leq 0.05$ were defined as differentially expressed proteins as previously defined (19).

Protein identification using tandem MS/MS mass spectrometry. For protein identification using tandem MS was performed following the previously used protocol (19). In brief, differentially expressed protein spots were excised from the post silver stained gels after DIGE. Trypsin digested peptides were extracted from the gel plugs with ultra-sonication. The peptides were separated using an Ultimate 3000 nano liquid chromatography system (LC Packings; Dionex) coupled to a HCTultra ion trap mass spectrometer (Bruker Daltonics, Leipig, Germany) equipped with an online nanospray source. The peptides were detected in the positive ion mode and fragmented by collision-induced dissociation. Precursor selection was set as $300-1,500 \mathrm{~m} / \mathrm{z}$. Two most abundant precursor ions were selected for MS/MS. Three scans were averaged to obtain an MS/MS mass spectrum.

MS/MS Ion Search was performed using generated an mgf file format obtained through individual online submission on the Matrixscience website (http://www.matrixscience.com). Rattus was selected as the primary taxonomy in the SwissProt (AA) database. For negative identification, a homology search using Rodentia (Rodents) was performed. Trypsin was designated as the enzyme and only one missed cleavage was allowed. Carbamidomethylation of cysteines was set as fixed modification and oxidation of methionine residues as variable modification. The mass tolerances were 0.6 Da for both protein (MS) and peptide (MS/MS). For all mass lists, no restrictions were applied for both the protein isoelectric point and molecular weight and ESI-TRAP was selected as instrument type. The proteins were considered identified when the peptide ion score was above the threshold value (typically $>25$, beyond green shading) in the Peptide score distribution. To minimize false positive results, only proteins with at least three non-duplicated peptides with significant hits were considered.

Statistical analysis. The data was averaged and presented as the mean $\pm \mathrm{SD}$. A repeated measures ANOVA was conducted to compare optic nerve injury grading and RGC density in different time points after pONT. All measurements at four distances and four quadrants from each animal were included in the overall ANOVA model, and the effects of quadrants and distances were controlled by multi-factors ANOVA model. When comparisons of RGC density among groups were performed within each quadrant, all measurements at four distances from each animal were included in the ANOVA model and the distance effect was controlled by multi-factors ANOVA model. One-way ANOVA followed by Tukey's test was applied for the analysis of differences in RGC degeneration between experimental and contralateral eyes were analyzed by paired t-test. $\mathrm{P}<0.05$ was considered statistically significant. All statistical analyses were performed using SPSS version 16 (IBM Corporation, Armonk, NY, USA).

\section{Results}

Primary and secondary RGC axonal damage after pONT. The optic nerve of one eye was partially transected while the other eye was used as control. A schematic diagram shows the location of pONT (Fig. 1A). As shown in Fig. 1B, each section was divided manually into two areas: Transected and non-transected regions (arrows) for axonal injury analysis (Fig. 1B). The grading of optic nerve injury in transected and non-transected regions at 8 weeks was compared to those at 1 week and the controls. For the transected region, the averaged grading at both time points was 5 (severe degeneration). Fig. 1C demonstrates that the injury in the non-transected region was substantial compared to the contralateral control at both time points $(\mathrm{P}=0.007$ at 1 week and 0.004 at 8 weeks $)$ and the grading was significantly increased from 1.45 at 1 week $(\mathrm{n}=8)$ to 1.8 at 8 weeks after $\mathrm{pONT}(\mathrm{P}=0.048 ; \mathrm{n}=12)$.

Primary and secondary RGC somal loss after pONT. To quantify the extent of primary and secondary loss of RGC bodies, the whole retinas collected at 1 and 8 weeks after 
A

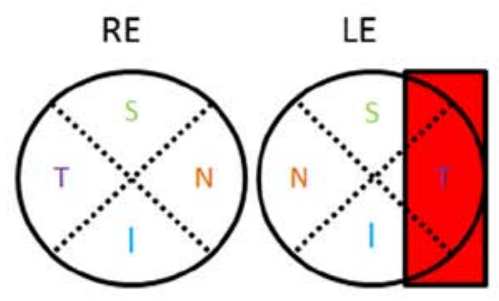

B

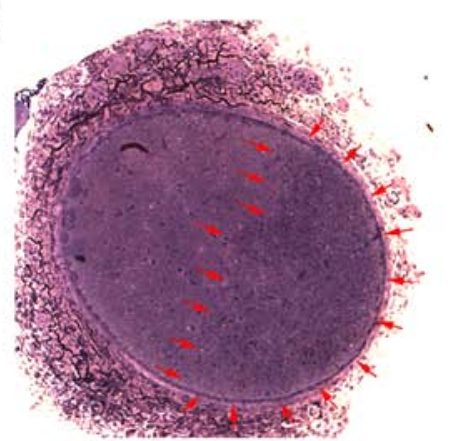

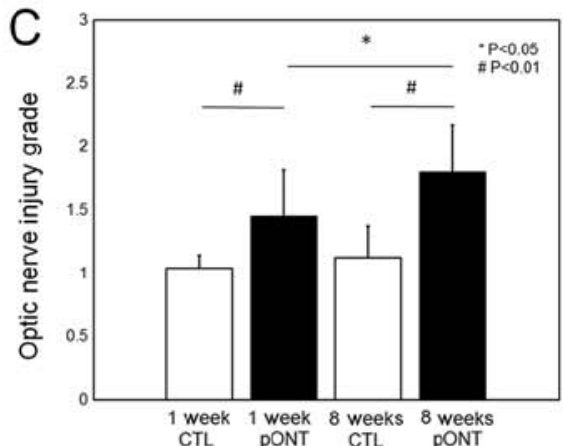

Figure 1. Semi-quantification of optic nerve injury after pONT. (A) Schematic diagram of pONT procedures in adult rats. A rectangle indicates the area transected by the diamond knife with the depth set at $0.28 \mathrm{~mm}$, which corresponds to approximately one third of the optic nerve diameter. (B) Micrograph of optic nerve cross section 2 weeks after pONT. Area of transected optic nerve region filled up with degenerated axons was manually circled as indicated by arrows. Both areas of transected (temporal) and non-transected (nasal) optic nerve were analyzed by optic nerve injury grading. (C) Analysis of optic nerve injury in non-transected region. Axonal damage of the optic nerve was graded on a scale of 1 to 5 (Grade: $1=$ no injury; $5=$ severe injury). The injury in the non-transected (nasal) region was significant at 1 week and 8 weeks, when compared to the control. The injury was significantly increased from 1 to 8 weeks ${ }^{\#} \mathrm{P}<0.01$ and ${ }^{*} \mathrm{P}<0.05$ as indicated. RE, right eye; LE, left eye; S, superior; N, nasal; I, inferior; T, temporal; CTL, control; pONT, partial optic nerve transection.
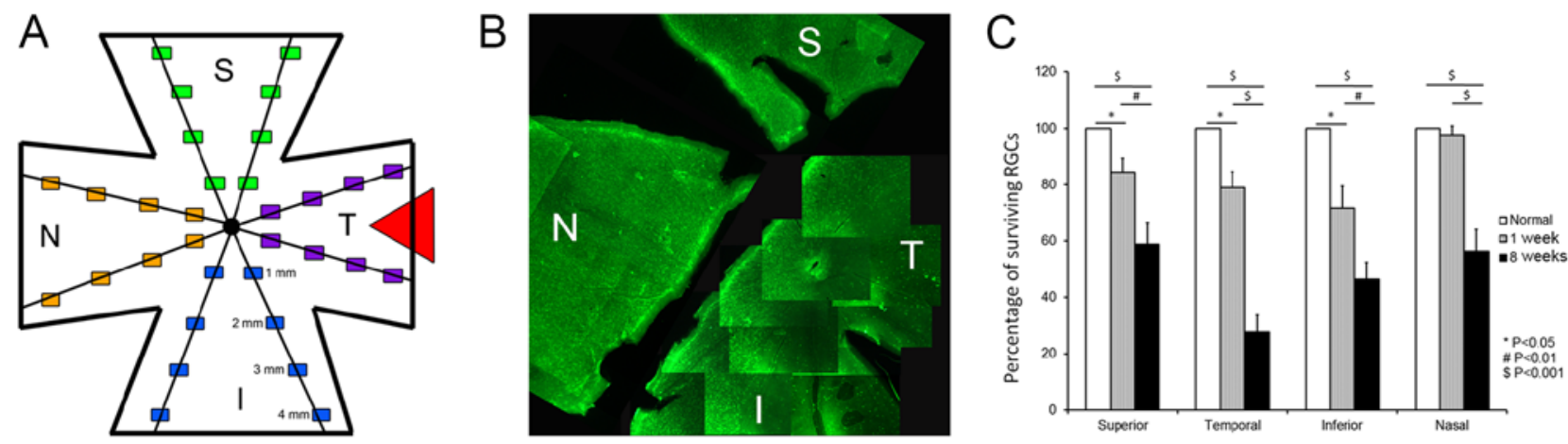

Figure 2. Quantitative analysis of surviving RGCs after pONT. (A) Sampling for topographic counting of RGC bodies on retinal whole mounts after immunohistochemistry using Rbpms antibody. Triangle indicates the site of pONT. (B) Representative composite fluorescence micrograph showing regional loss of Rbpms-labeled RGC bodies on retinal flat mount 8 weeks after pONT. The loss of RGCs in the temporal quadrant was remarkable, while partial RGC loss was observed in superior and inferior quadrants. (C) Patterns of RGC body loss after pONT. There was significant loss of RGC bodies in temporal, superior, and inferior quadrants was noted at one week after pONT compared to control. However, there was no significant RGC body loss in the nasal quadrant. At 8 weeks after pONT, the losses of RGC bodies in all retinal quadrants were significant when compared to control. The decreases in the numbers of surviving RGCs in all retinal quadrants at 8 weeks was significant when compared to corresponding regions at 1 week. ${ }^{"} \mathrm{P}<0.01,{ }^{\mathrm{S}} \mathrm{P}<0.001$ and ${ }^{*} \mathrm{P}<0.05$ as indicated. $\mathrm{S}$, superior; $\mathrm{N}$, nasal; I, inferior; T, temporal; RGCs, retinal ganglion cells; pONT, partial optic nerve transection.

pONT were subjected to the established procedures of Rbpms immunohistochemistry, which is widely regarded as a specific RGC marker. Fig. 2A illustrates the sampling scheme for topographical quantification of RGC bodies. Fig. 2B shows the dramatic loss of Rbpms-labeled RGC bodies, whilst predominantly in the temporal region, it was also observed in parts of the superior and inferior retina at 8 weeks after pONT, with mild to moderate loss of RGC bodies in the nasal region and portions of the superior and inferior retina. The percentage loss of RGC bodies in the experimental eye compared to the corresponding retinal quadrant in the untreated control is shown in Fig. 2C. Approximately 14.6, 21.1 and 28.2\% reduction of RGC numbers in the superior, temporal, and inferior retinal quadrants respectively was noted at 1 week after pONT. These RGC losses were statistically significant, when compared to contralateral control (Fig. 2B; $\mathrm{P}=0.047$, 0.037, $0.024)$. In contrast, no noticeable change was observed in the nasal quadrant at this time point $(2.4 \% \mathrm{P}=0.65)$. At 8 weeks after pONT, the percentage losses at superior, temporal, inferior, and nasal quadrants were 41.28 $7.92,72.3 \pm 5.99$,
53.64 \pm 6.1 , and 43.63 $\pm 7.74 \%$ respectively (all P-values <0.001) when compared to controls. The losses of RGC bodies at all these four quadrants increased between 1 to 8 weeks $(\mathrm{P}<0.01)$. In summary, the retinal sample collected from the temporal quadrant was the primary site of RGC degeneration, while the nasal quadrant suffered later RGC loss during secondary RGC degeneration.

Regional protein expression after pONT. To determine the protein changes associated with development of secondary loss of RGCs, the retinas at two weeks after pONT were collected. To target the mechanisms of primary and secondary RGC degeneration, each retina was divided into 4 retinal quadrants and proteins from each quadrant were extracted and analyzed separately by DIGE. The protein profile of each retinal quadrant of experimental eye was compared to their corresponding retinal quadrant in the contralateral control eye. In total, 24 proteins were found to be increased or decreased by more than $20 \%$ (Fig. 3; t-test, $\mathrm{P}<0.05$ ) at two weeks after pONT. The identities and fold changes of these regulated proteins were 

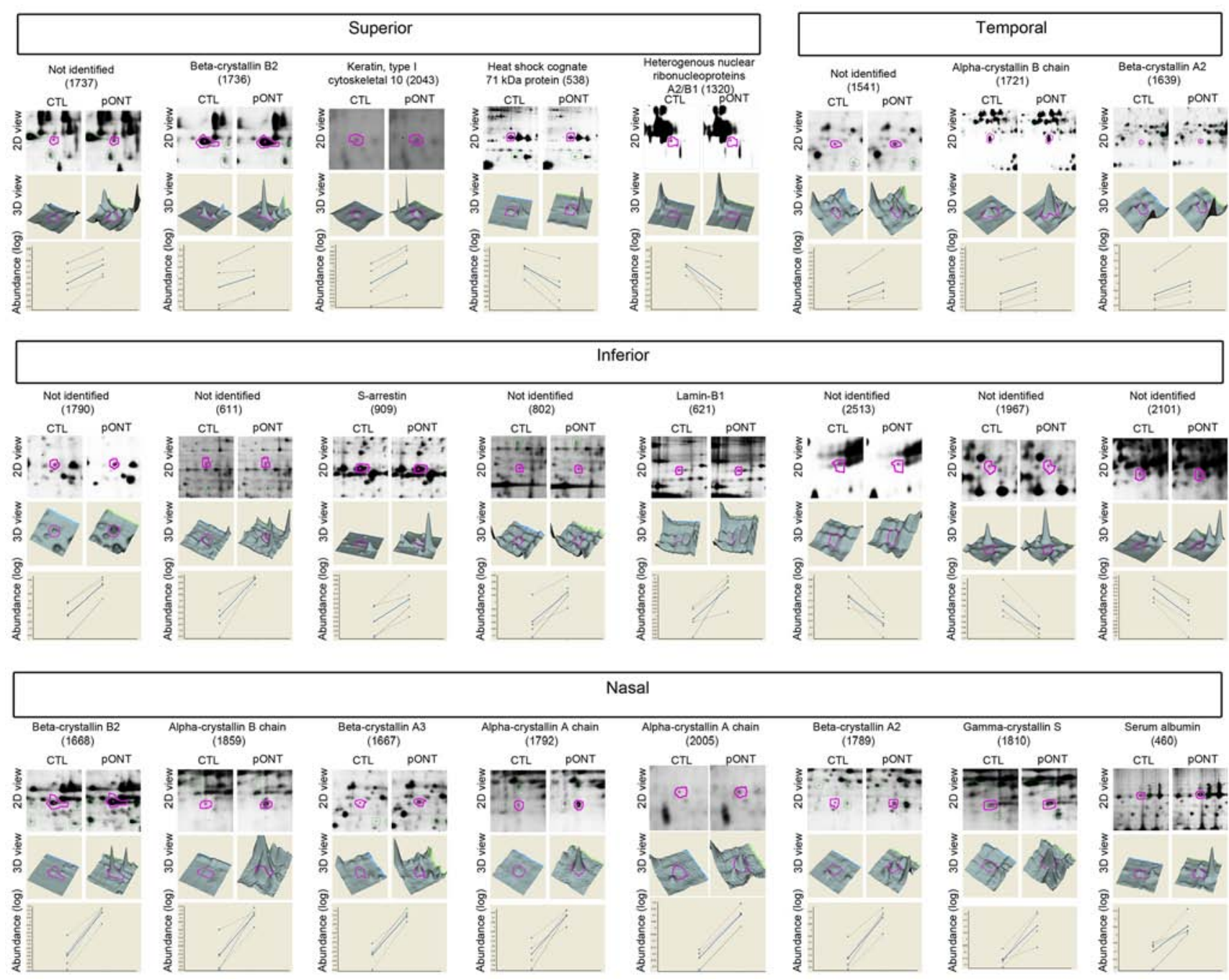

Figure 3. Differential expression of 24 proteins in superior, temporal, inferior, and nasal retinal quadrants at 2 weeks after pONT identified by tandem mass spectrometry. 2D and 3D views of individual in-gel protein spots in CTL and after pONT are shown. In the gels shown, the protein spot was detected (circled) by the DeCyder Differential Analysis software and subjected to protein identification. The name of each protein and in-gel spot number was listed. For protein quantification, the standardized log abundance of the protein spot (y-axis) was plotted against CTL and retinas after pONT (x-axis). Solid lines represent averaged group expression with $>1.2$ fold change and $\mathrm{P}<0.05$ while dotted lines denote individual expression ( $\mathrm{n}=4$ ). $2 \mathrm{D}$, two dimensional; 3D, three dimensional; CTL, control; pONT, partial optic nerve transection.

determined by tandem mass spectrometry and listed in Table I. Eight proteins were recorded as 'unidentified', mainly due to poor MS signals and low peptide abundance. There were 4, 2, 2 and 8 protein spots representing differentially expressed proteins in superior, temporal, inferior and nasal quadrant respectively. The two-dimension (2D) view and 3-dimension (3D) view of these regulated proteins in the gel are shown in Fig. 3. The measurement of standardized abundance of each sample (dotted line; $\mathrm{n}=4$ ) was recorded and averaged (solid line) for comparison. Upregulation of $\alpha$-crystallin B chain (P23928) and $\beta$-crystallin A2 (Q9JJV1) was observed in both temporal and nasal quadrants, with a higher fold change occurring in the nasal quadrant ( $\alpha$-crystallin B chain $=3.51$ fold; $\beta$-crystallin $\mathrm{A} 2=2.13)$ than the temporal $(\alpha$-crystallin $\mathrm{B}$ chain $=1.42$ fold; $\beta$-crystallin A2=1.23) quadrant. More $\beta$-crystallin B2 upregulation was observed in the nasal (3.84 fold) than the superior (1.27-fold) quadrant. Two gel spots corresponding to the same $\alpha \mathrm{A}$ crystallin chain (P24623) were found in the nasal quadrant. Among the four sets of comparisons, 10 out of 16 identified spots were found to be crystallins, belonging to six crystallin members. Other identified spots were keratin (type I cytoskeletal 10; Q61FW6), heat shock cognate $71 \mathrm{kDa}$ protein (P63018), S-arrestin (P15887), lamin-B1 (P70615), and serum albumin (P02770).

\section{Discussion}

Direct injury to the optic nerve leads to RGC axonal damage at the initial lesion site and body loss, followed by widespread loss in the neighboring area. The present study divided the retinal sample into portions and observed primary and secondary loss of RGCs after pONT in these quadrants and explored potential protein candidates associated with primary and secondary RGC degeneration. In particular, using proteomic analysis (DIGE) and protein identification (tandem MS), it was possible to demonstrate differential regulation of retinal proteins in the area (nasal region) adjacent to the primary injury site (temporal region) two weeks after pONT, by which time the RGC bodies have not disappeared. The present finding indicates a dynamic regulation of crystallin proteins in both over 


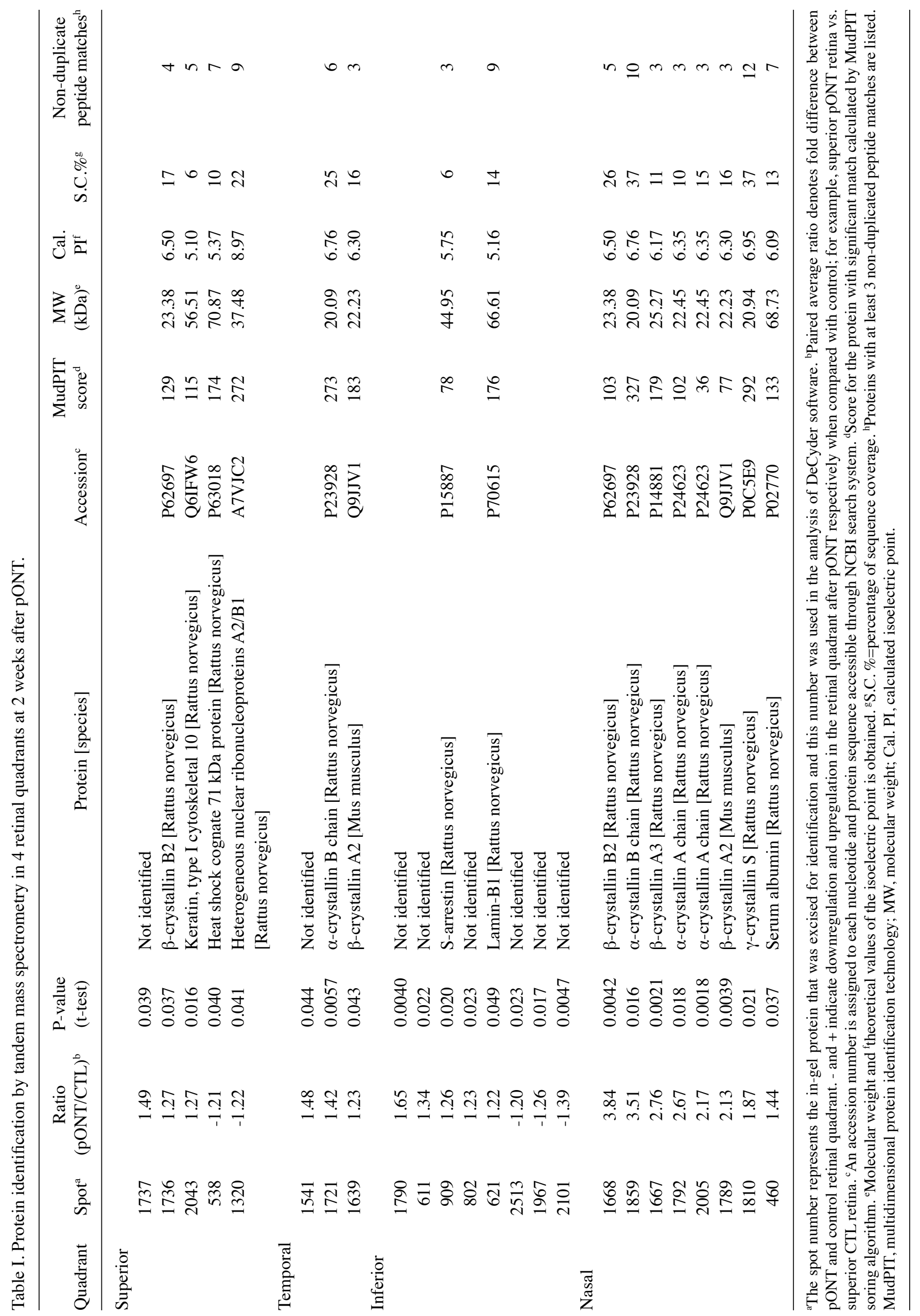


timely and topographically. Previous studies performed by the team have determined that $\alpha \mathrm{A}$ and $\alpha \mathrm{B}$ provides pro-survival effects to RGCs against optic nerve injury $(26,27)$. The current novel data, further support the hypothesis that not only the injured neurons react to injury, but also the intact RGCs in neighboring neuronal tissue respond to the insult and probably initiate self-defense function $(28,29)$.

Primary and secondary RGC degeneration can be clearly differentiated in the retina and optic nerve at times up to 8 weeks after pONT. Histologically, the demarcation line between two types of RGC degeneration is easily seen in the retinal whole-mount immunostained with Rbpms antibody and in the optic nerve cross-section stained with toluidine blue. For the level of retina, approximately 72, and 44\% of RGCs were lost at primary (temporal) and secondary (nasal) RGC degeneration sites respectively at 8 weeks after pONT. This is consistent with the findings of Levkovitch-Verbin et al (11) who reported approximately 41 and $35 \%$ fewer RGCs in the primary RGC degeneration zone (superior) and secondary degeneration zone (inferior) respectively 9 weeks after pONT, but $63 \%$ loss in the primary zone at 8 days, other studies showing a greater extent of RGC body loss in the primary injury site than the secondary zone. The discrepancy between the percentages of RGC loss may be due to different RGC labeling techniques. Delivery of Fluorogold to the superior colliculus on both sides only pre-labels the RGCs with axons projecting to superior colliculi and a higher percent of labeling requires proficient surgical skill. In addition, the shrunken retrogradely labeled cells that are not morphologically RGC-like would be excluded. On the other hand, whether the expression of Rbpms proteins and intensity of immunolabeling is altered by different types of injuries should also be addressed. Our previous study demonstrated that Rbpms is a specific marker for RGCs under various adverse conditions such as NMDA-induced excitotoxicity, optic nerve axotomy and experimental glaucoma with IOP elevation. The use of an RGC marker is considered useful for quantifying RGC in disease states $(20,21)$ which is supported by its use in many studies (30-33). Therefore, we believe this model, combined with careful dissection is suitable for exploring potential protein candidates involved in secondary mechanisms.

The present study characterized the differential regulation of retinal proteins in relation to their locations with significant loss of RGC bodies and axons after pONT. In contrast, optic nerve axotomy is known to result in an acute loss of RGCs. In rodents, the complete axotomy of the optic nerve causes over $90 \%$ of RGCs to disappear in 2 weeks (34). The mechanisms leading to total RGC degeneration have been widely studied. A few of researchers have established the procedures of pONT in rats, which provide for spatial separation of primary from secondary degeneration $(8,11,35-38)$, allowing investigation of the mechanism of secondary RGC degeneration. This model is useful to assess the sequences of events contributing specifically to secondary degenerative events in both RGC body and axons, and is relevant to the optic nerve degeneration in some ocular diseases, such as glaucomatous, traumatic, and ischemic optic neuropathy (15). Commencing $5 \mathrm{~min}$ to 3 days after pONT, early events, including increased expression of manganese superoxide dismutase, and decreased catalase activity, and calcium accumulation, lead to the over production of ROS and changes in mitochondrial morphology and function $(13,39)$. After several days to months, there is an infiltration of macrophages and activation of microglia, which are involved in secondary RGC degeneration, while swelling in myelinated axons and myelin sheath thickening causes the following visual dysfunction $(39,40)$. However, the biochemical data may not be able to determine the pathways of primary and secondary degeneration if the entire retina is analyzed. Chiha et al (41) initiated studies to separate the injured and non-injured retina for microarray and their results indicated complex primary injury gene regulation and delayed response in two different locations. To the best of our knowledge, this report is the first to demonstrate the regional protein regulation in the retina after pONT and, in particular, that stress proteins are selectively induced in the region for secondary RGC degeneration.

Collectively, we have demonstrated the upregulation of a group of crystallin proteins at a non-injured location rather than the injured area at two weeks after pONT. The upregulated crystallin proteins included $\alpha \mathrm{A}, \alpha \mathrm{B}, \beta \mathrm{A} 2, \beta \mathrm{A} 3$, and $\beta \mathrm{B} 2$. These proteins generally function as chaperones and protect cells. Coincidently, our previous study found that the genes for these crystallin members are predominantly expressed in the RGC layer cells and to a lesser extent, in the inner nuclear layer cells (26). It is tempting to link the increased expression of crystallin proteins with the number of surviving RGCs and their protective response to stress. The expression levels of crystallins vary with the nature of insult and degree of damage such as intraocular pressure elevation (26) and complete optic nerve axotomy (27). This explains why $\alpha \mathrm{B}$ and $\beta \mathrm{A} 2$ crystallin were increased approximately to 1.4and 1.2-fold in the injured region, compared with 3.5- and 2.1-fold in the non-injured region. Our experimental design allowing location-specific tissue sampling facilitates study of local regulation of proteins. In addition, the simultaneous upregulation of both $\alpha$ and $\beta$ crystallin protein suggests a common mechanism regulating the expression of these genes and proteins in the retinas $(28,29)$. The temporal and spatial regulation of crystallin genes are believed to be modulated by different arrangements of developmentally regulated transcription factors such as Pax-6, ROR $\alpha$, and heat shock factor 2 and 4 , and other transcription factors. $\beta / \gamma$ crystallins are implicated in RGC axonal regeneration through an autocrine, inflammation-induced, or astrocyte-derived CNTF-mediated mechanism. The upstream signaling of crystallins induction leading to RGC survival and regeneration of healthy and intact neurons should be further investigated.

Our study has limitations in that the exact role of each protein has not been determined, but it does provide fundamental data to understand how RGC degeneration progresses. An increased level of serum albumin in the non-injured region (nasal) may be due to one or a combination of the following sources: i) retinal vasculature, ii) vitreous, or iii) de novo synthesis within the retina. In normal conditions, the blood-retina barrier provides tight junctions between the endothelial cells and retinal pigment epithelium. It is possible that there is a local breakdown in the blood-retina barrier as a result of primary RGC degeneration or synthesis of intracellular albumin in response to localized oxidative injury similar to that reported experimental glaucoma in monkey (42). In the 
current study, a mixed protein response was observed in the superior and inferior regions. Protein expression of keratin type I, S-arrestin, and Lamin-B1 was increased, whereas heat shock cognate $71 \mathrm{kDa}$ protein and heterogeneous nuclear ribonucleoproteins A2/B1 were downregulated. The protein changes at these two regions may reflect the dramatic alteration in structures and RNA/protein synthesis at the transition zone between primary and secondary degeneration but this is yet to be confirmed.

The primary injury in the CNS leads to neuronal loss at the initial lesion site and widespread loss in the neighboring area. In the optic nerve, traumatic and ischemic insults cause the RGCs to die progressively, which involves secondary degeneration. Although numerous studies have demonstrated direct neuroprotective and neuroregenerative ability of $\alpha$ and $\beta$ crystallins (43-48), little is known about the stress response and self-defense mechanism of crystallins in the neighboring areas next to the injury site. The present proteomics analysis revealed specific protein regulation in the portion of the retina corresponding to the transected and non-transected optic nerve. The majority of regulated proteins are crystallin family members. Whether this regional induction of crystallins functions to protect neurons against the noxious effects from primary injury requires further investigation.

\section{Acknowledgements}

The authors would like to thank Dr Joseph Caprioli (University of California Los Angeles, Los Angeles, USA) for providing helpful discussion and Dr Maureen Valerie Boost (Hong Kong Polytechnic University, Hong Kong, P.R. China) for her diligent proofreading of the article.

\section{Funding}

The present study was supported by the Henry G Leong Endowed Professorship Fund (to CHT), General Research Funds [grant nos. BQ46K(CHT), PolyU 251006/14M (TCL) and PolyU5605/13M(HHLC)], PolyU Internal Grants [grant nos. G-YBBU(CHT), G-YBBS(HHLC), G-YBGS(HHLC), Z-0GF(HHLC), G-YBHJ(CWD), G-SB26(CWD), G-YBQX(TCL) and G-YBXH(TCL)] and the Gerald Oppenheimer Family Foundation (to JMKK).

\section{Availability of data and materials}

The datasets used and/or analyze during the current study are available from the corresponding author on reasonable request.

\section{Authors' contributions}

CL was a major contributor in proteomic approach and analysis, and contributed equally with JMKK to experimental design and all data analyses. KKL contributed to design, performed biochemical assays and proteomic experiments, analyzed and interpreted the proteomic data. HC, CWD and CHT contributed to experimental design and interpreted the proteomic data. JMKK contributed to conception and design, generation of animal model, acquisition of samples, analysis of RGC body and axonal degeneration, and was a major contributor in writing the manuscript. All authors read and approved the final manuscript.

\section{Ethics approval and consent to participate}

The use of animals in the study was approved by the Animal Research Committee of the University of California, Los Angeles. The procedures were performed in compliance with the ARVO Statement for the Use of Animals in Ophthalmic and Vision Research.

\section{Patient consent for publication}

Not applicable.

\section{Competing interests}

The authors declare that they have no competing interests.

\section{References}

1. Bazan NG, Rodriguez-deTurco EB and Allan G: Mediators of injury in neurotrauma: Intracellular signal transduction and gene expression. J Neurotrauma 12: 791-814, 1995.

2. Crowe MJ, Bresnahan JC, Shuman SL, Masters JN and Beattie MS: Apoptosis and delayed degeneration after spinal cord injury in rats and monkeys. Nat Med 3: 73-76, 1997.

3. Dusart I and Schwab ME: Secondary cell death and the inflammatory reaction after dorsal hemisection of the rat spinal cord. Eur J Neurosci 6: 712-724, 1994.

4. Faden AI: Pharmacological treatment of central nervous system trauma. Pharmacol Toxicol 78: 12-17, 1996

5. Liu D and McAdoo DJ: An experimental model combining microdialysis with electrophysiology, histology and neurochemistry for exploring mechanisms of secondary damage in spinal cord injury: Effect of potassium. J Neurotrauma 10: 349-362, 1993.

6. Quigley HA: Neuronal death in glaucoma. Prog Retin Eye Res 18: 39-57, 1999.

7. Quigley HA, Addicks EM, Green WR and Maumenee AE: Optic nerve damage in human glaucoma. II. The site of injury and susceptibility to damage. Arch Ophthalmol 99: 635-649, 1981.

8. Yoles E and Schwartz M: Degeneration of spared axons following partial white matter lesion: Implications for optic nerve neuropathies. Exp Neurol 153: 1-7, 1998.

9. Yoles E and Schwartz M: Potential neuroprotective therapy for glaucomatous optic neuropathy. Surv Ophthalmol 42: 367-372, 1998.

10. Levkovitch-Verbin H, Quigley HA, Kerrigan-Baumrind LA, D'Anna SA, Kerrigan D and Pease ME: Optic nerve transection in monkeys may result in secondary degeneration of retinal ganglion cells. Invest Ophthalmol Vis Sci 42: 975-982, 2001.

11. Levkovitch-Verbin H, Quigley HA, Martin KR, Zack DJ, Pease ME and Valenta DF: A model to study differences between primary and secondary degeneration of retinal ganglion cells in rats by partial optic nerve transection. Invest Ophthalmol Vis Sci 44: 3388-3393, 2003.

12. Davis BM, Guo L, Brenton J, Langley L, Normando EM and Cordeiro MF: Automatic quantitative analysis of experimental primary and secondary retinal neurodegeneration: Implications for optic neuropathies. Cell Death Discov 2: 16031, 2016.

13. Fitzgerald M, Bartlett CA, Harvey AR and Dunlop SA: Early events of secondary degeneration after partial optic nerve transection: An immunohistochemical study. J Neurotrauma 27: 439-452, 2010.

14. Levkovitch-Verbin H, Dardik R, Vander S and Melamed S: Mechanism of retinal ganglion cells death in secondary degeneration of the optic nerve. Exp Eye Res 91: 127-134, 2010

15. Li HY, Ruan YW, Ren CR, Cui Q and So KF: Mechanisms of secondary degeneration after partial optic nerve transection. Neural Regen Res 9: 565-574, 2014.

16. Levkovitch-Verbin H, Spierer O, Vander S and Dardik R: Similarities and differences between primary and secondary degeneration of the optic nerve and the effect of minocycline. Graefes Arch Clin Exp Ophthalmol 249: 849-857, 2011. 
17. Li HY, Hong X, Huang M and So KF: Voluntary running delays primary degeneration in rat retinas after partial optic nerve transection. Neural Regen Res 14: 728-734, 2019.

18. O'Hare Doig RL, Chiha W, Giacci MK, Yates NJ, Bartlett CA, Smith NM, Hodgetts SI, Harvey AR and Fitzgerald M: Specific ion channels contribute to key elements of pathology during secondary degeneration following neurotrauma. BMC Neurosci 18: 62, 2017.

19. Wu Y, Lam CS, Tse DY, To CH, Liu Q, McFadden SA, Chun RK, Li KK, Bian J and Lam C: Early quantitative profiling of differential retinal protein expression in lens-induced myopia in guinea pig using fluorescence difference two-dimensional gel electrophoresis. Mol Med Rep 17: 5571-5580, 2018.

20. Kwong JM, Caprioli J and Piri N: RNA binding protein with multiple splicing: A new marker for retinal ganglion cells. Invest Ophthalmol Vis Sci 51: 1052-1058, 2010.

21. Kwong JM, Quan A, Kyung H, Piri N and Caprioli J: Quantitative analysis of retinal ganglion cell survival with Rbpms immunolabeling in animal models of optic neuropathies. Invest Ophthalmo Vis Sci 52: 9694-9702, 2011.

22. Ishii Y, Kwong JM and Caprioli J: Retinal ganglion cell protection with geranylgeranylacetone, a heat shock protein inducer, in a rat glaucoma model. Invest Ophthalmol Vis Sci 44: 1982-1992, 2003.

23. Jia L, Cepurna WO, Johnson EC and Morrison JC: Patterns of intraocular pressure elevation after aqueous humor outflow obstruction in rats. Invest Ophthalmol Vis Sci 41: 1380-1385, 2000.

24. Lam TC, Li KK, Lo SC, Guggenheim JA and To CH: Application of fluorescence difference gel electrophoresis technology in searching for protein biomarkers in chick myopia. J Proteome Res 6: 4135-4149, 2007.

25. Lam TC, Li KK, Lo SC, Guggenheim JA and To CH: A chick retinal proteome database and differential retinal protein expressions during early ocular development. J Proteome Res 5 : 771-784, 2006

26. Piri N, Song M, Kwong JM and Caprioli J: Modulation of alpha and beta crystallin expression in rat retinas with ocular hypertension-induced ganglion cell degeneration. Brain Res 1141: 1-9, 2007.

27. Munemasa Y, Kwong JM, Caprioli J and Piri N: The role of alphaA- and alphaB-crystallins in the survival of retinal ganglion cells after optic nerve axotomy. Invest Ophthalmol Vis Sci 50 3869-3875, 2009.

28. Piri N, Kwong JM and Caprioli J: Crystallins in retinal ganglion cell survival and regeneration. Mol Neurobiol 48: 819-828, 2013.

29. Piri N, Kwong JM, Gu L and Caprioli J: Heat shock proteins in the retina: Focus on HSP70 and alpha crystallins in ganglion cell survival. Prog Retin Eye Res 52: 22-46, 2016.

30. Sharma TP, Liu Y, Wordinger RJ, Pang IH and Clark AF: Neuritin 1 promotes retinal ganglion cell survival and axonal regeneration following optic nerve crush. Cell Death Dis 6: e1661, 2015.

31. Wang W, Chan A, Qin Y, Kwong JMK, Caprioli J, Levinson R, Chen L and Gordon LK: Programmed cell death-1 is expressed in large retinal ganglion cells and is upregulated after optic nerve crush. Exp Eye Res 140: 1-9, 2015.

32. Obara EA, Hannibal J, Heegaard S and Fahrenkrug J: Loss of Melanopsin-expressing retinal ganglion cells in severely staged glaucoma patients. Invest Ophthalmol Vis Sci 57: 4661-4667, 2016.

33. Joachim SC, Renner M, Reinhard J, Theiss C, May C, Lohmann S, Reinehr S, Stute G, Faissner A, Marcus K, et al: Protective effects on the retina after ranibizumab treatment in an ischemia model. PLoS One 12: e0182407, 2017.

34. Clarke DB, Bray GM and Aguayo AJ: Prolonged administration of NT-4/5 fails to rescue most axotomized retinal ganglion cells in adult rats. Vision Res 38: 1517-1524, 1998.
35. Fitzgerald M, Payne SC, Bartlett CA, Evill L, Harvey AR and Dunlop SA: Secondary retinal ganglion cell death and the neuroprotective effects of the calcium channel blocker lomerizine. Invest Ophthalmol Vis Sci 50: 5456-5462, 2009.

36. Chu PH, Li HY, Chin MP, So KF and Chan HH: Effect of lycium barbarum (wolfberry) polysaccharides on preserving retinal function after partial optic nerve transection. PLoS One 8: e81339, 2013

37. Li HY, Ruan YW, Kau PW, Chiu K, Chang RC, Chan HH and So KF: Effect of Lycium barbarum (Wolfberry) on alleviating axonal degeneration after partial optic nerve transection. Cell Transplant 24: 403-417, 2015.

38. Li H, Liang Y, Chiu K, Yuan Q, Lin B, Chang RC and So KF: Lycium barbarum (wolfberry) reduces secondary degeneration and oxidative stress, and inhibits JNK pathway in retina after partial optic nerve transection. PLoS One 8: e68881, 2013.

39. Payne SC, Bartlett CA, Harvey AR, Dunlop SA and Fitzgerald M: Myelin sheath decompaction, axon swelling, and functional loss during chronic secondary degeneration in rat optic nerve. Invest Ophthalmol Vis Sci 53: 6093-6101, 2012.

40. Payne SC, Bartlett CA, Savigni DL, Harvey AR, Dunlop SA and Fitzgerald M: Early proliferation does not prevent the loss of oligodendrocyte progenitor cells during the chronic phase of secondary degeneration in a CNS white matter tract. PLoS One 8: e65710, 2013.

41. Chiha W, LeVaillant CJ, Bartlett CA, Hewitt AW, Melton PE, Fitzgerald $\mathrm{M}$ and Harvey AR: Retinal genes are differentially expressed in areas of primary versus secondary degeneration following partial optic nerve injury. PLoS One 13: e0192348, 2018.

42. Carter-Dawson L, Zhang Y, Harwerth RS, Rojas R, Dash P Zhao XC, WoldeMussie E, Ruiz G, Chuang A, Dubinsky WP and Redell JB: Elevated albumin in retinas of monkeys with experimental glaucoma. Invest Ophthalmol Vis Sci 51: 952-959, 2010.

43. Ying $\mathrm{X}$, Zhang J, Wang $\mathrm{Y}$, Wu N, Wang $\mathrm{Y}$ and Yew DT: Alpha-crystallin protected axons from optic nerve degeneration after crushing in rats. J Mol Neurosci 35: 253-258, 2008.

44. Wu N, Yu J, Chen S, Xu J, Ying X, Ye M, Li Y and Wang Y: $\alpha$-Crystallin protects RGC survival and inhibits microglial activation after optic nerve crush. Life Sci 94: 17-23, 2014.

45. Böhm MR, Prokosch V, Brückner M, Pfrommer S, Melkonyan H and Thanos $\mathrm{S}$ : $\beta \mathrm{B} 2$-crystallin promotes axonal regeneration in the injured optic nerve in adult rats. Cell Transplant 24: 1829-1844, 2015.

46. Shao WY, Liu X, Gu XL, Ying X, Wu N, Xu HW and Wang Y: Promotion of axon regeneration and inhibition of astrocyte activation by alpha A-crystallin on crushed optic nerve. Int J Ophthalmol 9: 955-966, 2016

47. Anders F, Teister J, Liu A, Funke S, Grus FH, Thanos S, von Pein HD, Pfeiffer N and Prokosch V: Intravitreal injection of $\beta$-crystallin B2 improves retinal ganglion cell survival in an experimental animal model of glaucoma. PLoS One 12: e0175451, 2017.

48. Wang YH, Yin ZQ and Wang Y: Synergistic effect of olfactory ensheathing cells and alpha-crystallin on restoration of adult rat optic nerve injury. Neurosci Lett 638: 167-174, 2017.

This work is licensed under a Creative Commons Attribution-NonCommercial-NoDerivatives 4.0 International (CC BY-NC-ND 4.0) License. 